\title{
Physicists question model of the Universe
}

Cosmologists gathered in London last month to voice their concerns over the current 'standard' model of the Universe. "There is a sense of desperation," says participant Douglas Scott, from the University of British Columbia in Vancouver. "The standard model is horribly ugly, but the data support it."

For most in the field, that desperation stems from two unexplained ingredients in the standard model: dark matter, thought to help ordinary matter clump together to form galaxies; and dark energy, invoked to explain the observation that the expansion of the universe is accelerating.

But physicists are increasingly questioning the model itself. Richard Lieu, from the University of Alabama in Huntsville, is one of those with

doubts. He says he organized the meeting, at Imperial College London, "for scientists to come forward and present any misgivings they may have about the standard model", although he invited speakers from all sides of the debate.

A broad consensus over the standard model has emerged over the past decade. It is

"The standard model is based on diverse lines horribly ugly." of evidence, such as measurements of the radiation left over from the early Universe (known as the cosmic microwave background), the distribution of galaxies and the brightness of distant supernovae.

Scientists at the meeting were asked to re-examine assumptions made when these observations were analysed. For example, Subir Sarkar, from the University of Oxford, UK, argued that data from the Wilkinson Microwave Anistropy Probe (WMAP) - the NASA satellite that measured the cosmic microwave background with unprecedented precision - make sense without dark energy if density perturbations in the early Universe had a different pattern to that usually assumed, and if our patch of the Universe differs from other parts. This explanation might seem more complicated than the standard model, says Sarkar, but he counters that invoking dark energy is "a profound problem from the viewpoint of fundamental physics".

Attendees were also asked to question the actual data. Speakers pointed out oddities in WMAP's maps of the microwave sky, such as the alignment of patterns on different scales along what has been cheekily dubbed an 'axis of evil', and the presence of more variation in the southern hemisphere than in the northern. Such unlikely effects could just be statistical quirks, but they seed uncertainty about how the measurements are being interpreted.

For the time being, proponents of the standard model say that they have yet to see a compelling theoretical alternative, but encourage those people who pursue them. "I doubt that anyone left that meeting convinced about some new idea," says Scott. "But having one's mind opened a little bit is healthy." Jenny Hogan 